\title{
Mutationism and the Dual Causation of Evolutionary Change
}

\author{
Arlin Stoltzfus \\ Center for Advanced Research in Biotechnology, 9600 Gudelsky Drive, Rockville, MD 20850
}

Biochemical Science Division, National Institute of Standards and Technology, Gaithersburg, MD, 20899-8310

email: arlin.stoltzfus@nist.gov

telephone: 2403146208 


\section{Abstract}

The rediscovery of Mendel's laws a century ago launched the science that William Bateson called "genetics", and led to a new view of evolution combining selection, particulate inheritance, and the newly characterized phenomenon of "mutation". This "mutationist" view clashed with the earlier view of Darwin, and the later "Modern Synthesis", by allowing discontinuity, and by recognizing mutation (or more properly, mutation-and-altereddevelopment) as a source of creativity, direction, and initiative. By the mid- $20^{\text {th }}$ century, the opposing Modern Synthesis view was a prevailing orthodoxy: under its influence, "evolution" was redefined as "shifting gene frequencies", i.e., the sorting out of pre-existing variation without new mutations, and the notion that mutation-and-altered-development can exert a predictable influence on the course of evolutionary change was seen as heretical. Nevertheless, mutationist ideas re-surfaced: the notion of mutational determinants of directionality emerged in molecular evolution by 1962, followed in the 1980's by an interest among evolutionary developmental biologists in a shaping or creative role of developmental propensities of variation, and more recently, a recognition by theoretical evolutionary geneticists of the importance of discontinuity and of new mutations in adaptive dynamics. The synthetic challenge presented by these innovations is to integrate mutation-and-altered-development into a new understanding of the dual causation of evolutionary change - a broader and more predictive understanding that already can lay claim to important empirical and theoretical results — and to develop a research program appropriately emphasizing the emergence of variation as a cause of propensities of evolutionary change. 


\section{Introduction}

From the recognition, a century ago, of the generality of mutation and particulate inheritance, emerged a view of evolution known today as "mutationism". According to many accounts (e.g., Berry 1982; Ridley 1985; Dawkins 1987, p. 305), the mutationists — led by such eminent geneticists as De Vries, Bateson, and Morgan — did not understand selection and treated evolution as a succession of genetic mutations. Mutationist ideas strongly influenced evolutionary biology early in the $20^{\text {th }}$ century, but fell form favor by mid-century, when the “Modern Synthesis" captured the central place it maintains today (Hull 2002).

Nevertheless, while the historical demise of mutationism is real, its scientific defeat is dubious. If one probes more deeply, to discover what mutationism represents, why mutationist ideas gained in popularity upon the discovery of genetics, and why the same ideas were opposed by evolutionists more faithful to Darwin, it becomes clear that the mutationists provided an alternative perspective on evolutionary causation as necessary today as it was a century ago.

\section{Darwin's world: evolution without mutation}

To understand what mutationism represents, one must understand the role of variation in the theory that preceded it, which perhaps is more foreign than most readers will have appreciated. Given Darwin's assumptions that inheritance is blending (not particulate), that the germ-line is responsive to external conditions (not isolated), and that hereditary factors shift gradually every generation (not rarely and abruptly, from one stable state to another), it is physically impossible for a rare trait, having arisen by some process, and conferring a fitness advantage of (for 
example) $2 \%$, to be passed on to offspring by a stable non-blended hereditary factor, thus conferring on the offspring a $2 \%$ advantage, and for such a process to continue for thousands of generations, until the previously rare trait prevails. We may think of evolution in this way: Darwin did not.

Instead, Darwin's conception of evolution as an automatic process of adjustment to altered conditions depended on a rampant process of "fluctuation", aptly named "creeping variation" by Muller (1956), yielding abundant "infinitesimally small inherited modifications" in response to the effect of altered "conditions of life" on the "sexual organs" (Chs. 1, 2, 4 and 5 of Darwin 1859). Creeping variation was not rare and discrete, but shifted hereditary factors continuously and cumulatively each generation, producing visible effects in "several generations" (Ch. 1 of Darwin 1859). This evolutionary process is automatic, because the same "altered conditions of life" that compromise the adaptive status quo call forth fluctuation. While Darwin's view also invoked Lamarckian effects, the fluctuation-selection process of "natural selection" was recognized immediately as its mechanistic core. Only this core mechanism remains in the reformed "neo-Darwinian" view of Weismann and Wallace, which expunged Lamarckism and reinterpreted creeping variation as a spontaneous process.

Darwin's view that "natural selection can act only by the preservation and accumulation of infinitesimally small inherited modifications" and without any "great and sudden modifications" (Ch. 4 of Darwin 1859) was not the only possible view. He knew that discrete "sports" or mutants arise, and that their effects may be heritable. Not just critics, but colleagues such as Huxley and Galton (Orr and Coyne 1992; Gould 2002) believed that such abrupt effects play an important role in evolution, but Darwin held fast to natura non facit saltum ("nature does not take 
a leap"), consistently emphasizing "infinitesimal" or "insensible" modifications, and dismissing "sports" as monstrous and unimportant. Darwin's commitment to such doctrines seems to have proceeded in a top-down manner, from his judgment that evolution is an exquisitely adaptive process, to the kinds of mechanisms that would allow this behavior, as Gould (2002) suggests:

Darwin reasoned that natural selection can only play such a [creative or directional] role if evolution obeys two crucial conditions: (1) if nothing about the provision of raw materials — that is, the sources of variation — imparts direction to evolutionary change; and (2) if change occurs by a long and insensible series of intermediary steps, each superintended by natural selection — so that "creativity" or "direction" can arise by the summation of increments ... As long as variation only supplies raw material; as long as change accretes in an insensibly gradual manner; and as long as the reproductive advantages of certain individuals provide the statistical source of change; then natural selection must be construed as the directional cause of evolutionary modification.

These conditions are stringent; and they cannot be construed as vague, unconstraining, or too far in the distance to matter. In fact, I would argue that the single most brilliant (and daring) stroke in Darwin's entire theory lay in his willingness to assert a set of precise and stringent requirements for variation - all in complete ignorance of the mechanics of heredity. Darwin understood that if any of these claims failed, natural selection could not be a creative force, and the theory of natural selection would collapse. (p. 140)

Whether this daring set of conjectures about variation is satisfied is the issue that would come to divide Darwin's followers from their critics. 


\section{Genetics and the Eclipse of Darwin's Theory}

By the second decade of the $20^{\text {th }}$ century, it was clear that the new science of genetics supported neither Lamarck's process of adaptive variation, nor Darwin's process of creeping variation. Johannsen's famous experiments had shown that selection for size among varieties of beans can sort out large or small varieties, yet selection among plants of a single variety (a "pure line") produces no change, though fluctuations in size continue to appear. The resulting rejection of Darwin's mechanism of evolutionary change, though it is treated today as an inscrutable mystery, attributable to "sheer misunderstandings" (Cronin 1991, p. 23), "misconceptions" (Mayr 1994a), and the like (e.g., Berry 1982; Eldredge 2001, p. 67), said to provoke only "mirth" in the "modern mind" (Dawkins 1987, p. 305), is not mysterious at all, but simply follows from the generalization that heritable changes emerge, not by creeping variation, but by "mutation", which soon came to mean "gene mutation" or alteration to the individual gene (Muller 1923) as distinct from the karyotype effects underlying the evolutionary arguments of De Vries (1905). Skeptics continued to hope for evidence of creeping variation, but geneticists seized upon the implications of "mutation as the basis of evolution — as the material upon which natural selection works" (Punnett 1911, p. 139).

Contrary to popular belief, the resulting revolution in evolutionary thinking was not a rejection of selection, but a recognition of the "dual nature of the problem" of evolution (Morgan 1903). When Punnett argues (above) for mutation as the "basis" of evolution, he is not equating evolution with mutation, but arguing for mutations as "the material upon which selection works." Bateson referred to selection as "obviously" a "true cause" (Bateson 1894, p. 5). De Vries (1905, p. 6) referred to it as Darwin's "great principle which rules the evolution of organisms," though 
for De Vries, the predominant mode of selection was species selection, not individual selection.

What, then, explains the widespread belief that the mutationists "essentially did away with natural selection" (Ayala and Fitch 1997)? The answer hinges on the fact that "natural selection" often does not refer to the universal principle of selection, i.e., the principle by which biased differential reproduction can lead to shifts in frequency, and ultimately to the persistence of an initially rare form at the expense of an initially common competitor. For instance, Darwin insists (above) that "natural selection can act only by ... infinitesimally small inherited modifications" yet the principle of selection has no such restriction, applying equally to “infinitesimally small" or "great and sudden" modifications; to "indefinite" or "definite" (directed) variations; and to rare or ever-present variations. By longstanding tradition, then, the principle of selection is bundled with Darwin's conjectures about variation, and invoked as an external force, a creative principle, or a "mechanism of evolution" (Bell 1997). Indeed, the contemporary Encyclopedia of Evolution presents "natural selection" as a "theory of evolution" notable for its seemingly gratuitous restrictions on variation:

In evolution by natural selection, the processes generating variation are not the same as the process that directs evolutionary change. Variation is undirected... What matters is that the mutations are undirected relative to the direction of evolution ... Natural selection differs from most alternative theories of evolution in the independence between the processes that direct variation and that direct evolution ... Darwin's theory is peculiar in that evolution is not an extension of the mutational process. (Ridley 2002, p. 800)

By contrast, in the mutationist view, evolution is an extension of the mutational process. 


\section{The mutationist view}

The heterogeneous view represented in the works of Bateson (1894), de Vries (1905), Morgan (1916), Punnett (1911), Vavilov (1922), Shull (1936) and Goldschmidt (1940) may be called "mutationism", though the name seems to have been popularized by opponents of the extreme saltationism of De Vries and Goldschmidt. The core mechanistic idea of mutationism is that events of "mutation" - which in the evolutionary writing of the past typically implicates not only an unseen change in the germ line, but also its phenotypic manifestation, i.e., mutation-andaltered-development — introduce variants that face differential survival (selection). To varying degrees, the mutationists interpreted this two-step process to imply that mutation (mutation-andaltered-development) is a source of discontinuity, initiative, creativity and directionality in evolution. Since they also recognized selection, their view incorporated dual causation by internal (mutational and developmental) and external (selective) causes.

These ideas are illustrated briefly below, emphasizing the roles ascribed to mutation. One should not assume from this that overlapping or complementary roles for selection were denied: De Vries rejected any creativity of selection, yet identified it as "the one directing cause of the broad lines of evolution" (De Vries 1905, p. 7); Bateson did not deny the overall importance of selection, yet shunned selective explanations as speculations outside the reach of experiment; Morgan's arguments for selective causes of directional trends were repeated by Huxley (1942, p. 498) and effectively became part of the Modern Synthesis. 


\section{Discontinuity}

The mutationists adopted a range of positions on discontinuity or discreteness, excluding only the Darwinian extreme of relying wholly on "infinitesimal" changes. De Vries adopted an antithetical position emphasizing dramatic changes that create new species (with intra-specific variation and selection playing only a minor role). Bateson challenged "the crude belief that living beings are plastic conglomerates of miscellaneous attributes ... and that by Variation any of these attributes may be subtracted or any other attribute added in indefinite proportion" (Bateson 1894, p. 80). In pursuit of a less speculative approach to evolution, he published Materials for the Study of Variation, a detailed catalog of 886 cases of discontinuous variation (Bateson 1894); he planned a second volume on continuous variation but never completed it. Morgan argued that "even the smallest changes that add to or subtract from a part in the smallest measurable degree" may arise by mutation, and these are "the most probable variants that make a theory of evolution possible" (Morgan 1925, p. 129). The fully discretized view of the mutationists clashed with the incipient Modern Synthesis view in Punnett's (1930) Nature review of Fisher's The Genetical Theory of Natural Selection:

Throughout the book one gets the impression that Dr. Fisher views the evolutionary process as a very gradual, almost impalpable one, in spite of the discontinuous basis upon which it works. Perhaps this is because he regards a given population as an entity with its own peculiar properties as such, whereas for the geneticist it is a collection of individuals. 


\section{Initiative}

The view that events of mutation initiate evolutionary change contrasts with the view that evolutionary change is initiated by a change in environment. While Darwin's followers see a certain inevitability in adaptation, in which altered conditions elicit an automatic "response" (using available variation), the mutationists developed a stochastic view, in which there are many combinations of new mutants and external circumstances, and in which, to persist, a variant must find itself in suitable circumstances. Opponents caricatured this as the "lucky mutant" view (Mayr 1963, p. 101), but it was hardly an unsophisticated appeal to chance, as shown by Morgan's stunning grasp of the probability of acceptance of new mutations (see also Punnett 1911, p. 142):

If through a mutation a character appears that is neither advantageous nor disadvantageous, but indifferent, the chance that it may become established in the race is extremely small, although by good luck such a thing may occur rarely. It makes no difference whether the character in question is a dominant or a recessive one, the chance of its becoming established is exactly the same. If through a mutation a character appears that has an injurious effect, however slight this may be, it has practically no chance of becoming established. If through a mutation a character appears that has a beneficial influence on the individual, the chance that the individual will survive is increased, not only for itself, but for all of its descendants that come to inherit this character. (Morgan 1916, p. 187) 
In this view, the dynamics of evolution (adaptive or not) depend on mutation rates, because as Shull (1936, p. 140) argues, "a gene produced twice by mutation has twice as good a chance to survive as if produced only once" (see also Morgan 1925, p. 142).

\section{Creativity}

The mutationists held that "the function of natural selection is selection and not creation. It has nothing to do with the formation of new variation" (Punnett 1911). The creativity of mutationand-altered-development may be illustrated (in the extreme case) by Goldschmidt's concept of a “macromutation", akin to Bateson's concept of a shift between "positions of organic stability". If a variant toad arises with fully formed eyes in the roof of its mouth, such that it must open its mouth to see - as in the actual toad pictured on p. 97 of Dawkins (1996) - this is an extreme yet coordinated change, and a "macromutation" if it is heritable. If such a variant supplants the parental form or becomes a separate species, this is a non-Darwinian, saltational change in which creativity is due largely to mutation-and-altered-development. In the case of less dramatic transformations, the creative role of mutation-and-altered-development is correspondingly less dramatic. In spite of Goldschmidt's notorious belief that distinctive phenotypic transformations suggested major genetic reorganizations ("systemic mutations"), he insisted that the complexity of the underlying genetic change is not the decisive issue:

It does not make any difference whether a single macroevolutionary step is caused by a major change within the chromosomal pattern, [that is,] a systemic mutation, or by a special kind of gene mutation with generalized effect, if such is imaginable. The decisive point is the single change which affects the entire reaction system of the developing 
organism simultaneously, as opposed to a slow accumulation of small additive changes. (Goldschmidt 1940, p. 251)

\section{Directionality}

On a one-dimensional scale of fitness or adaptedness, every change is either "up" (beneficial) or "down" (deleterious), but in a multi-dimensional space of phenotypes, every change has a distinctive direction. In the book review cited earlier, Punnett chides Fisher for denying to mutations "any importance in determining the direction of evolutionary change". Most mutationists invoked mutation as a source of direction in considering features such as lepidopteran wing patterns (Punnett 1911, p. 145).

The views of mutationists on directionality emerge most clearly in regard to parallel evolution, which was assumed to indicate non-random tendencies of variation, since "it strains one's faith in the laws of chance to imagine that identical changes should crop out again and again if the possibilities are endless and the probabilities equal" (Shull 1935, p. 448). Vavilov noted that the same varieties or polymorphisms often occur in parallel, even in distantly related species in the same genus or family, and he argued for a causal role of this "law of homologous variation" (Vavilov 1922) in parallel evolution. As an example, Vavilov reports that lentils (Ervum lens), a food crop, and vetch (Vicia sativa), a weed, have many homologous variations, and notes that vetches sometimes mimic lentils so closely in cultivated fields that their seeds cannot be separated by mechanical sorters:

the role of natural selection in this case is quite clear. Man unconsciously, year after year, by his sorting machines separated varieties of vetches similar to lentils in size and 
form of seeds, and ripening simultaneously with lentils. The same varieties certainly existed long before selection itself, and the appearance of their series, irrespective of any selection, was in accordance with the laws of variation. (Vavilov 1922, p. 85)

\section{Dual causation}

A final distinctive feature of mutationism is the mechanistic and explanatory dualism apparent in the above quotation, invoking a causal role both for selection, and for the character or dynamics of mutation-and-altered-development. While Darwin's view might be said to share this dualism in the sense that both fluctuation and selection are required for change as opposed to non-change, Darwin's followers emphasize that selection, while not sufficient to cause change by itself, is the proper cause of the manner of change, that is, its directionality, dynamics, creativity, and so on (e.g., as in the quotation from Ridley above). For instance, Darwin preceded Vavilov in recognizing a principle of "analogous variation" due to a similar "inherited constitution", but denied it any influence on evolutionary change:

But characters thus gained [by analogous variation] would probably be of an unimportant nature, for the presence of all important characters will be governed by natural selection, in accordance with the diverse habits of the species, and will not be left to the mutual action of the conditions of life and of a similar inherited constitution. (ch. 5, Darwin 1859)

\section{The Modern Synthesis emerges from the "gene pool"}

Darwin's view of evolution as an automatic process of adaptation to altered conditions seemed to require abundant, uniform, and infinitesimal variation, yet mutations seemed to be rare, 
idiosyncratic, and discrete. The key to reconciling the two, as recognized in 1926 by Chetverikov, is the notion that every species has a "gene pool" that soaks up variation like a “sponge" (Chetverikov 1997), "maintaining" it for later use by selection, and ensuring an abundance of minute heritable variations in every trait, in every generation, as Darwin had conjectured. The "Modern Synthesis" between genetics and Darwinism thus denies a direct link between mutations and evolutionary change, claiming instead that evolution begins with the "gene pool".

Accordingly, the source of initiative in this view is not the occurrence of mutations - which are individually insignificant (Dobzhansky et al. 1977, p. 72) and merely "replenish the supply of variability in the gene pool" (Stebbins 1966, p. 29) — but the change in conditions that brings on selection of variation already present (e.g., Dobzhansky 1955, p. 282; Mayr 1963, p. 613; Dobzhansky et al. 1977, p. 6; e.g., Stebbins 1982, p. 160). Thus "evolution is not primarily a genetic event. Mutation merely supplies the gene pool with genetic variation; it is selection that induces evolutionary change" (Mayr 1963, p. 613).

As in Darwin's view, the sole "direction-giving force” is natural selection (Mayr 1980), which chooses from the abundance of directions represented in the "gene pool" (e.g., Dobzhansky 1955, p. 282; Mayr 1963, p. 101; Dobzhansky et al. 1977, p. 6, 72; Mayr 1994a), whose buffering capacity insulates evolution from mutational dynamics:

Mutations are rarely if ever the direct source of variation upon which evolutionary change is based. Instead, they replenish the supply of variability in the gene pool which is constantly being reduced by selective elimination of unfavorable variants ... 
Consequently, we should not expect to find any relationship between rate of mutation and rate of evolution. (Stebbins 1966, p. 29)

Given that the "gene pool" was seen to satisfy Darwin's requirements for variation, the architects of the Modern Synthesis identified natural selection - which they compared to a poet, a composer, a sculptor, and even William Shakespeare - as the creative force in evolution (Gould 1977).

This view became so deeply engrained that "evolution" was redefined for most purposes as "the conversion of intra-population variation into inter-population variation in space and time" (Lewontin 1965), or "shifting gene frequencies", so that the mutational introduction of noveltythe crux of mutationism - is no longer part of the process of "evolution", but is subsumed within a precondition (i.e., intra-population variation). Mutationism came to be seen as discredited:

The genetic work of the last four decades has refuted mutationism (saltationism) so thoroughly that it is not necessary to repeat once more all the genetic evidence against it. (Mayr 1960, p. 355)

if ever it could have been thought that mutation is important in the control of evolution, it is impossible to think so now (Ford 1971, p. 361)

As late as 1932 T.H. Morgan was asserting that 'natural selection does not play the role of a creative principle in evolution', but ten years later all but a very few biologists were agreed on an evolutionary theory based firmly on Darwin's own ideas knitted with subsequent developments in genetics. (Berry 1982, p. 14) 
for simplicity we speak of mutation as the first stage in the Darwinian process, natural selection as the second stage. But this is misleading if it suggests that natural selection hangs about waiting for a mutation which is then either rejected or snapped up and the waiting begins again. It could have been like that: natural selection of that kind would probably work, and maybe does work somewhere in the universe. But as a matter of fact on this planet it usually isn't like that. (Dawkins 1996, p. 87)

\section{Mutationist themes in contemporary research}

How was mutationism eliminated in favor of the Modern Synthesis? To distinguish these two views, it is not sufficient to show that selection occurs in nature or that some changes are small, since mutationism also allows for selection and for small effects. Instead, to distinguish these two views, which both invoke selection and mutation, one must address how these components causally determine the manner in which evolutionary change occurs, that is, the observed or expected behavior of evolving systems in regard to the issues that I denote here as creativity, discontinuity, directionality and initiative (dynamics), and that Mayr, Ford and Berry (above) refer to in terms of creativity, saltation, and control. The architects of the Modern Synthesis offered theoretical and empirical arguments on all of these issues, yet mutationist views have reemerged importantly in molecular evolution, theoretical evolutionary genetics, and evolutionary developmental biology.

\section{Molecular evolution}

The "mistaken idea that mutations can establish the rate and direction of evolution" (Stebbins 1982, p. 79) re-emerged in the 1960's, when GC:AT biases in mutation were invoked to account 
for systematic differences in DNA and protein composition (Freese 1962; Sueoka 1962).

Molecular evolutionists commonly assume that the relative probability of an evolutionary change has two factors, one reflecting the rate of mutational origin, and another reflecting the probability of fixation, a view that follows directly from formal models (Kimura 1983), and that provides an explicit basis for statistical methods of sequence analysis (Goldman and Yang 1994; Muse and

Gaut 1994). While few molecular evolutionists have noted the connection between this view and mutationism (Nei 1987), many focus their research on the role of mutation bias in shaping genes, proteins and genomes (Beletskii and Bhagwat 1996; Petrov and Hartl 1999; Fryxell and Zuckerkandl 2000; Singer and Hickey 2000; Yampolsky and Stoltzfus 2005). Such effects are not trivial: they must be taken into account when inferring phylogenies (Mooers and Holmes 2000) or testing for positive selection (Dagan et al. 2002); and they are arguably more important than effects of fitness in accounting for propensities of amino acid change (Yampolsky and Stoltzfus 2005).

Nevertheless, while an enormous body of circumstantial evidence suggests that propensities of evolutionary change reflect propensities of mutation, the interpretation of this evidence has been held hostage to doubts about neutral evolution, on the assumption that to accept variation-biased evolution is to accept neutral evolution. However, this assumption is not necessary, as argued by J.L. King in attempting to a explain a pattern — an observed correlation between the frequencies of amino acids in proteins and their numbers of codons in the genetic code - that he and Jukes had attributed initially to neutral evolution:

In an earlier article, we wrote that, in the case of adaptive evolution, "one particular amino acid will be optimal at a given site in a given organism, and it matters little whether there 
are six possible codons (as there are for serine) or only one (as there is for methionine)." On further reflection, we must reject this deterministic view of adaptive molecular evolution ... Suppose that, at a given time, there are several possible amino acid substitutions that might improve a protein, and among these are changes to serine and to methionine; serine, with its six codons, has roughly six times the probability of becoming fixed in evolution. Once this has occurred, the mutation to methionine may no longer be advantageous. Ultimately, the amino acid frequency composition of proteins will reflect the frequencies at which the various amino acids arise by mutation (King 1971, p. 89)

\section{Evolutionary genetics}

The architects of the Modern Synthesis employed both empirical and theoretical arguments in support of infinitesimalism and against discontinuity. The empirical argument for infinitesimalism took the form of i) showing that differences between closely related species are composed of infinitesimal differences and ii) extrapolating from such close comparisons to all evolved differences. The problem is that the facts do not allow the first step. In their landmark review of these arguments, Orr and Coyne (1992) conclude that there is "little evidence for the neo-Darwinian view", and that "mutations of large effect are sometimes important", conclusions that still stand (Orr 2005).

In his theoretical argument for infinitesimalism, Fisher (1930) argued that, in attempting to improve a microscope by shifting or reshaping its parts, smaller adjustments would more likely be beneficial. From a geometric version of this argument, he concluded that the probability of beneficial effect reaches an upper limit of $1 / 2$ for a mathematically infinitesimal change. Though this argument was influential (Orr 2005), Orr and Coyne (1992) suggest that it is "weak" due to 
its lack of a basis in biology or population genetics. By combining a variant of Fisher's model with a model of fixation of new mutations, Orr $(1999 ; 2005)$ concludes that the size distribution of changes during adaptation is exponential, favoring small effects yet with large effects contributing substantially, particularly in the early stages of adaptation.

A related model of adaptation was the subject of a recent study hailed as "the first empirical test of an evolutionary theory" (Bull and Otto 2005). Rokyta, et al. (2005) carried out one-step adaptive walks with bacteriophage $\phi X 174$, and compared the results to a theoretical model. The model (Orr 2002) assumes that the chance of taking an adaptive step $i$ is proportional to $\mu_{i} \pi_{i}\left(\mu_{i}\right.$, mutation rate to an allele with probability of fixation $\pi_{i}$ ) and more specifically, given the simplifying assumptions that $\mu$ is uniform and that $\pi_{i} \approx 2 s_{i}$, that this chance is proportional to $s_{i}$. Experimental results led to two revisions of this model, the first concerning a more exact formula for the probability of fixation. More importantly, Rokyta, et al. find that the chance of an adaptive step is influenced by mutational effects - including a 12-fold transition:transversion bias and differences of up to 3-fold in the multiplicity of mutational paths to alternative amino acid states (an effect of the genetic code as in King's argument above) - so strongly that their results “are 21 times more likely under the mutation-adjusted version of Orr's model than under the original model", which assumed uniform mutation. That is, this "first empirical test of an evolutionary theory" was not a test of the "shifting gene frequencies" theory of adaptation, but of the "new mutations" theory, and the results reveal a predictable influence of mutational propensities on adaptation.

The dynamics of new mutations also emerge in regard to the theoretical argument against internal causes of directionality that cleared the way for the Modern Synthesis view that 
selection is the only source of direction in evolution (Huxley 1942, p. 56; Mayr 1960, p. 355; Simpson 1967, p. 159; Gould 2002, p. 509). As Fisher (1930) and Haldane (1932) argued, in a one-locus model with a forward:reverse bias in rates of mutation from allele $\underline{b}$ to $\underline{B}$, the bias in mutation will not result in fixation of $\underline{B}$ in a $\underline{b}$ population if $\underline{B}$ is even slightly deleterious, because selection will push the frequency of $\underline{B}$ downward much more strongly than mutation pushes it upward. This result was interpreted broadly to reduce the whole issue of direction to a winner-takes-all contest between opposing pressures: mutation rates are small, therefore selection beats mutation, and "The whole group of theories which ascribe to hypothetical physiological mechanisms, controlling the occurrence of mutations, a power of directing the course of evolution, must be set aside . . The sole surviving theory is that of Natural Selection" (Fisher 1930, p. 20).

Nevertheless, Yampolsky and Stoltzfus (2001) show that a bias in mutation can bias the course of adaptation in a mutationally favored direction, even when selection coefficients are large and mutation rates are small. In their 2-locus 2-allele model, an initial ab population can evolve to one of two beneficial alternatives, $\underline{\mathrm{Ab}}$ or $\underline{\mathrm{aB}}$, representing isolated adaptive peaks; $\underline{\mathrm{Ab}}$ is the more fit of the two by a factor $\mathrm{K}=\mathrm{s}_{\underline{\mathrm{Ab}}} / \mathrm{s}_{\underline{\mathrm{B}}}$, but $\underline{\mathrm{aB}}$ is favored by a bias in mutation of magnitude $\mathrm{B}=$ $\mu_{\underline{a b}-\underline{a B}} / \mu_{a b}-\underline{A b}$. When $\mathrm{B}>\mathrm{K}$, the mutationally favored peak prevails. The key to this effect is that, because the variant genotypes are not present initially, the bias in mutation is a bias in rates of origin, so that the joint probability of origin-fixation is enhanced by a higher rate of origin. By contrast, the Fisher-Haldane model treats mutation as a surrogate for selection (a mass-action "pressure" that might cause fixation), not as the process that introduces novelty. 
Thus, theoretical evolutionary genetics seems to have developed with a blind spot in regard to new mutations, a situation that is only now being corrected (for precursors, see Hill 1982; Gillespie 1984; Mani and Clarke 1990). For instance, in order to evaluate the prospects for Wright's "shifting balance" mode of evolution, Phillips (1996) begins by adding a previously missing "phase 0" consisting of "waiting for a compensatory mutation". Hartl and Taubes (1998) argue that classical theory is "not informative about the central evolutionary problem of the origin, progression and limit of adaptation" because of its inadequate treatment of mutation:
Almost every theoretical model in population genetics can be classified into one of two major types. In one type of model, mutations with stipulated selective effects are assumed to be present in the population as an initial condition ... The second major type of models does allow mutations to occur at random intervals of time, but the mutations are assumed to be selectively neutral or nearly neutral.

This blind spot is not accidental. The architects of the Modern Synthesis, eager to resurrect Darwinian themes and antagonistic to mutationism, conceded only that mutation is "ultimately necessary", erected the "gene pool" as a buffer between mutation and "evolution", and denied any specific influence of mutation-and-altered-development on directionality and dynamics. This parochial view was formalized, and drummed into the heads of several generations of students, by re-defining "evolution" as the sorting out of pre-existing variation, so that the causal "pressures" or "forces" of "evolution" may be defined as mass-action forces that shift frequencies. Ostensibly, these "forces" include "mutation", but they do not include a discrete process that introduces novelty, which is what the mutationists meant by "mutation". 


\section{Evolutionary Developmental Biology}

In the early 1980's, "evo-devo" began to assert itself as a distinct discipline with an unorthodox appreciation for Bateson (homeotic mutations) and Goldschmidt (phenocopies, reaction norms). Today the mainstream of "evo-devo" is focused on reconstructing evolutionary changes in developmental mechanisms. Nevertheless, a minority view insists that "integrating development into evolutionary theory" involves something more profound, as in the suggestion that "NeoDarwinian theory can predict what is more likely to survive once it has appeared, but it cannot address the question of what is more likely to be generated." (Alberch 1982, p. 314), or the claim of Thomson (1985, p. 222) that:

The whole thrust of the developmentalist approach to evolution is to explore the possibility that asymmetries in the introduction of variation at the focal level of individual phenotypes, arising from the inherent properties of developing systems, constitutes a powerful source of causation in evolutionary change

Thus, while the mainstream challenge of evo-devo is to overlay phylogenies with a chronicle of inferred changes in ontogeny, the revolutionary strain of evo-devo aims to promote, as an evolutionary cause, a role of developmental processes in transformation, i.e., mutation-andaltered-development, or what Arthur (2000) calls "developmental reprogramming".

This idea requires some clarification. One may distinguish a strong (i.e., bold) and a weak (i.e., unremarkable) claim for evolutionary effects of developmental biases or asymmetries in variation. The weak claim is that an absolute bias - the distinction between possible and impossible variants - may cause evolution to prefer some directions to others. The strong claim 
(Thomson 1985; Yampolsky and Stoltzfus 2001; Arthur 2004) is that analogous effects arise more commonly by mere quantitative asymmetries, that is, more versus less preferred transformations.

The weak claim, usually invoked in the language of "developmental constraints" (Maynard Smith et al. 1985), is weak because it is non-controversial, being accepted across the spectrum of evolutionary thought (Eimer 1898; Mayr 1963, p. 608), for the obvious reason that one has no choice but to accept that a developmentally impossible change is an evolutionarily impossible change. Though logically necessary, this feckless notion is based on an absolute distinction that renders it both biologically unlikely and experimentally intractable: one cannot, in a finite period of time, prove that a conceivable variant cannot be produced, and this necessarily leaves open the possibility that its non-occurrence in evolution (which has far more time to sample possibilities) is due to deleterious fitness effects, a possibility that cannot be tested without producing the variant that has not yet been produced.

By contrast, the strong claim is both tractable and controversial. Propensities of variation can be measured, and compared to evolutionary propensities; if there is any question as to whether a bias among observed variants is due to fitness effects, the variants can be tested for their relative fitness (though this is difficult). The strong claim is controversial because of the widespread belief, arising from the Fisher-Haldane argument (above), that mere quantitative biases of variation are ineffectual or non-causal. For instance, in their seminal review of "developmental constraints", Maynard Smith and others (1985) cite the Fisher-Haldane argument explicitly without contradicting its "opposing pressures" logic, which they apparently accept; in response to their suggestion of important evolutionary consequences of a bias "more accessible" or "easily 
generated" forms, Reeve and Sherman (1993) raise the Fisher-Haldane objection: "why couldn't selection suppress an 'easily generated physicochemical process' if the latter were disfavored?"

Clearly, empirical studies are needed to characterize propensities of variation and relate them to propensities of evolutionary change. Two studies may be mentioned in this regard. Alberch and Gale (1985) characterized propensities of skeletal variation in the amphibian autopod (hand or foot) by treating developing autopods with colchicine, so as to produce aberrant autopods with missing digits or phalanges. This methodology avoids the problems posed by the rareness of mutations, and ensures that biases in the morphologies produced reflect potentialities of development, and not fitness effects (since the treated individuals all survive the treatment). The patterns of skeletal deviation observed in the laboratory for a salamander species and a frog species were compared taxon-specifically to patterns of evolutionary divergence (i.e., applying Vavilov's law of homologous variation). The results show a crude but clearly non-random correspondence between taxon-specific developmental preferences for digit and phalange loss, and taxon-specific patterns of digit and phalange loss in evolution, supporting the hypothesis that developmental propensities of variation cause propensities of evolutionary change.

A second example may be drawn, not from "evo-devo", but from contemporary quantitative genetics. The rate and direction of evolution in quantitative characters is jointly determined by the "G matrix" of additive genetic variances and covariances, and by fitness differentials (Steppan et al. 2002). Mathematically, the two are factors to be multiplied, so there is no formal reason to privilege one as a "cause" while describing the other as a "constraint". The remarkable demonstration by Schluter (1996) of a natural case in which adaptive divergence takes place in 
the multivariate direction of greatest additive genetic variance is prima facie evidence of a shaping influence of propensities of variation. This conclusion is not inevitable, however: when only adult variation is measured, the effects of propensities of variation are confounded with effects of past generations of selection, and of selection on pre-adult stages in the current generation. Such effects could be untangled by methods for determining the contribution of new mutations to G (Houle et al. 1996; Houle 1998).

\section{Integrating mutation-and-altered-development into evolutionary}

\section{theory}

A century after the discovery of mutation, we have yet to integrate mutation and altered development into our understanding of evolutionary causation. Due to the manner in which evolutionary theory has passed through the narrow conceptual bottleneck of the Modern Synthesis, we lack a recognized, general causal linkage between the introduction of variants by mutation-and-altered-development and the course of evolution, that is, a linkage that - as implied by certain theoretical models, and suggested by empirical results reviewed abovegoes beyond "constraints", and allows that propensities of variation cause propensities of evolution.

The preceding kinds of claims - about the lack of recognition of certain kinds of causes, or the inadequacy of certain theories to accommodate these causes - are bound to be controversial, therefore a more explicit argument is appropriate. In constructing such an argument, I will focus on Thomson's conjecture, or the strong claim of evolutionary developmental biology, that developmental biases in the introduction of variation are a "powerful source of causation in 
evolutionary change", presumably by shaping divergence and contributing to parallelisms, trends, and so on. From the above presentation, there should be no doubt that this kind of causation, whether powerful or weak, is not currently part of the Modern Synthesis, because internal causes of direction are denied, e.g., Mayr (1980) defines the Modern Synthesis as "the theory that selection is the only direction-giving factor in evolution."

Nevertheless, though the Modern Synthesis currently excludes Thomson's conjecture, perhaps Mayr's interpretation is unnecessarily narrow, so that the theory could be reinterpreted to accommodate this possibility.

To demonstrate that this is not the case, that is, to demonstrate that the kind of developmental causation invoked in Thomson's conjecture cannot be incorporated into the Modern Synthesis without contradiction, I will argue that the Modern Synthesis is too narrow to accommodate, as the simplest possible well formulated invocation of this kind of causation, the role of development in King's argument cited earlier. The developmental process at work implicitly in King's argument is a complex multi-stage process involving literally hundreds of RNAs and proteins. The outcome of this process of transcription and translation is encapsulated for practical purposes in a mapping of amino acid phenotypes to nucleotide-triplet genotypes: the genetic code. Relative to other developmental processes, the developmental expression of triplet nucleotides as amino acids is unusual in its lack of plasticity and pleiotropy: a triplet genotype is developmentally expressed by its modal amino acid roughly $99.99 \%$ of the time (i.e., the error rate for translation is roughly $10^{-4}$ ), and although the three nucleotide loci that encode an amino acid interact epistatically, they are not pleiotropic (i.e., each nucleotide, in collaboration with two others, influences only a single amino acid phenotype). 
In King's argument, it is crucial that this mapping of phenotypes to genotypes imposes asymmetries on the introduction of variant amino acids, an effect that plays a subsidiary role in the experiments of Rokyta, et al. For instance, replacing Methionine with Isoleucine at position 416 in the F gene of $\phi \mathrm{X} 174$ is a beneficial change that can be accomplished by three different mutations from the Methionine codon ATG to an Isoleucine codon (ATC, ATA or ATT), whereas other beneficial replacements can be accomplished by only a single mutation (Table 1 of Rokyta et al. 2005). In King's argument, the sum of all such effects is that the chance for an amino acid phenotype to arise by mutation-and-altered-development (nucleotide mutation followed by transcription and translation) is proportional to its number of codons in the genetic code; the evolutionary consequence of this bias is a steady-state distribution in which amino acid frequencies are proportional to the numbers of codons assigned in the genetic code.

Maynard Smith (1975) also recites King's argument, concurring with its conclusion, and commenting that

if we accept the selectionist view that most substitutions are selective, we cannot at the same time assume that there is a unique deterministic course for evolution. Instead, we must assume that there are alternative ways in which a protein can evolve, the actual path taken depending on chance events. This seems to be the minimum concession the selectionists will have to make to the neutralists; they may have to concede much more (p. 105)

Note that, for Maynard Smith, the role of mutation is a "chance event", consistent with Modern Synthesis doctrine (Mayr 1983), whereas King's argument relies on a non-uniformity in the effects of mutations to account for an observed correlation. Maynard Smith states that the 
correlation "can be explained in strictly selectionist terms" (p. 105), by which he means that it is consistent with selective allele fixations. Yet since both authors agree that the same correlation would arise through random fixations, it is only by mixing up proximate causes (the mode of fixation) with ultimate causes (a bias in the introduction of amino acid variants by mutation-and-altered-development) that one can attribute the correlation to the mode of fixation.

Molecular evolutionists may invoke "mutation pressure" or "mutation-driven evolution" (Li 1997) in such a case, but this also fails to provide an appropriate causal explanation, since the bias does not reside in genetic mutation (but in its phenotypic effects) and is not due to an effect of mass-action "pressure" on allele frequencies (but to a bias in the novelty-introducing process). One might suggest that the genetic code imposes "constraints" on evolution, but this is either i) not a causal explanation, if "constraint" means merely a factor with no understood mechanism of causation, or ii) not a correct explanation, if "constraint" means a limit that prevents some types of amino acid changes - as Maynard Smith (1983) notes elsewhere, there are "no constraints on the kinds of changes that can arise in DNA sequences." One might suggest that the "opportunism" of selection results in a kind of "first come, first served" effect in adaptation, but since the same effect would be seen for random fixations, this so-called "opportunism" is not a property of selection or adaptation, but of the origin-fixation process generally.

This is not to say that King's argument cannot be described: the correlation is caused by an asymmetric developmental mapping of amino acids to codons that imposes a corresponding set of asymmetries on the introduction of variant amino acid states by mutation and altered development (Thomson's “powerful source of causation”); regardless of whether variants reach 
fixation by selection or drift, it is assumed that any biases imposed by differential reproduction are orthogonal factors that leave these asymmetries unperturbed.

That is, King's argument can be described, but not without either i) using conventional ideas of causation that result in false attributions, or ii) introducing heterodox concepts that contradict established doctrines, including the Fisher-Haldane argument (above), the doctrine that variation is "random" and does not favor one kind of outcome over another (Ridley 1985), the doctrine that selection is the source of non-randomness and "ultimate source of explanation in biology" (Ayala 1970), and the doctrine that development cannot be construed as an evolutionary cause (Maynard Smith 1983; Mayr 1994b). Presumably, this is precisely why the significance of King's argument has been lost to history — molecular evolutionists still associate the hypothesis of variation-biased evolution with neutral evolution (e.g., Galtier and Lobry 1997).

\section{A framework for integration}

To formalize the challenge of integrating mutation-and-altered-development into evolutionary theory, it is helpful to begin with Sober's (1984) concept of "source laws" and "consequence laws" as applied to selection. Theoretical population genetics provides consequence laws for selection that allow one to compute the precise distribution of outcomes expected under various conditions, given the differences in fitness among variants present in a population; the source laws for selection would provide a means to compute fitness differences from descriptions of phenotypes and their environments. While we lack a general mechanistic theory to generate source laws for fitness, limited source laws can arise from sub-theories (e.g., case-specific optimality models) and useful heuristics (e.g., dividing fitness into "components" of survival, mating and fecundity). Joining source laws and consequence laws within a larger analytical 
framework connects the causes of fitness differences with their expected evolutionary consequences, allowing a predictive theory.

By analogy, then, the problem of integrating mutation into evolutionary theory is the problem of identifying source laws and consequence laws that can be united so as to connect the mutational and developmental causes of propensities of variation — or at least a phenomenological description of such propensities — with their expected evolutionary consequences.

With this distinction in mind, it becomes clear that, although Medawar suggested that "the main weakness of modern evolutionary theory is its lack of a fully worked out theory of variation, that is, of candidature for evolution" (Medawar 1967, p. 104), the deficiency involves both source and consequence laws. In the Modern Synthesis, there is apparently a single source law for the emergence of new variants, repeated in every textbook, namely that this process is "random" (by contrast, Darwin had an elaborate theory of variation expressed in two volumes on the subject). The only consequence law is that, on the rare occasions when variation is absent, there is a "constraint" or "limit" that prevents "selection" from ensuring an optimum (Barton and Partridge 2000). As explained above, the lack of such consequence laws in the Modern Synthesis is an explicit denial and not an accidental omission. If our aim is to integrate mutation-and-altereddevelopment into evolutionary theory, a different theory is needed.

As a general framework, one may begin with the conception of Vrba and Eldredge (1984) of evolution as a process of the introduction and sorting of variants in a hierarchy of reproducing entities, where sorting refers to the cumulative effects of differential reproduction, either biased (selection) or unbiased (drift), and where the hierarchy may include genes, chromosomes, cells, organisms, species, and so on. Non-randomness in this process may arise from biases in the 
introduction of variants, and biases in the reproduction of variants (selection). This conceptual framework seems sufficiently broad to encompass an enormous set of cases. Within it, one may consider the source and consequence laws of variation, with particular attention to how such laws are determined, what they represent in terms of physical processes, and how they may be joined to generate testable predictions.

\section{Source laws of variation}

The source laws of variation come from genetics and development. Rather than imagining a list of all conceivable variants along with their relative probabilities, it is expedient to conceive of source laws (and consequence laws) in terms of relative biases or asymmetries favoring one class of variant relative to another (with added dimensions for effects of environment and genetic background - ignored here for the sake of simplicity). Such biases may be considered at various levels of organization, from the effect of mutations on nucleotide sequences and chromosome structures; to effects on expression, splicing, and protein structure; to metabolic pathways and regulons; to cell types and cell interactions; to tissues and organs; to morphologies and behaviors; and ultimately to the unitary dimension of fitness or adaptedness.

At the lowest level of effects on nucleotide sequence properties, mutation biases are well known. For instance, it is observed in both prokaryotic and eukaryotic systems that the "transition" mutations between chemically related nucleotides $(A \Leftrightarrow \mathrm{G}$ and $\mathrm{C} \Leftrightarrow \mathrm{T})$ are favored several-fold over "transversions." In mammals, due to an effect of methylation at $\mathrm{CpG}$ dinucleotides on DNA repair, transitions at such sites are favored a further 10-fold, and transversions, 4-fold (Hwang and Green 2004). Micro-deletions are often favored several-fold over micro-insertions, and their length distribution follows a power law (Zhang and Gerstein 2003). Our knowledge of 
the propensities of other types of mutation (duplications, large insertions and deletions, translocations, and so on) is meager.

One should not assume that biases in phenotypic variation must be explained by such mutation biases. The propensities of autopod skeletal deviation studied by Alberch and Gale (above) clearly emerge by some physical process, but since this process does not include genetic mutation, the proper locale of a causal explanation is in terms of developmental operators and not mechanisms of DNA damage and repair.

At the level of morphology, Alberch and Gale offer two conjectures regarding how to find, and how to interpret, developmental biases in variation. First, they suggest that the propensities of altered development that emerge in the context of environmental perturbations will tend to correspond to those that emerge in the context of genetic perturbation, i.e., mutation. Several observations may be cited in regard to this conjecture of what Ancel and Fontana (2000) call "plastogenetic congruence". Geneticists commonly encounter "phenocopies", individuals that lack a given mutant allele, yet exhibit the same mutant phenotype, due to environmental effects, or to a different mutation. A more systematic type of evidence is that, when variance-covariance matrices for quantitative traits are partitioned into genetic and environmental components, the two components tend to be correlated (Cheverud 1988). Finally, in computer models that use RNA folding as developmental system, plastogenetic congruence between the neighborhood of phenotypic variants accessible by plasticity and the neighborhood of phenotypic norms accessible by mutation is evident (Ancel and Fontana 2000).

To the extent that plastogenetic congruence holds generally (an issue of great practical importance), it will tend to ameliorate two problems with the classic method used by Darwin, 
Bateson, and Vavilov, which is to infer properties of the process of variation by direct observation of variants in wild or domesticated species. The first problem is that such nongenetic approaches do not reveal the extent to which observed effects are heritable: this problem diminishes in importance to the extent that heritable and non-heritable effects show the same propensities. Second, one does not know the extent to which the probability of observing a variant is due to past selection, as opposed to biases in the emergence of variation: but if plastogenetic congruence holds, one may emulate the method of Alberch and Gale for eliminating (or otherwise controlling) fitness effects.

The second conjecture of Alberch and Gale is that "changes in many genes affecting different morphogenetic parameters may result in the same morphological change" (Alberch and Gale 1985, p. 19). Stoltzfus (1999) gives a diverse set of examples of a similar concept of "systemic" biases in variation, in which the emergence of one type of molecular phenotype is favored over another because it represents a larger fraction of locally accessible genotype space. Such a principle, if generally applicable, could prove highly useful as a means to translate developmental propensities into volumes of genetic state-space, so as to link developmental source laws with consequence laws (see below).

With respect to quantitative characters, there is a growing body of work on the $\mathbf{G}$ matrix of additive genetic variances and covariances among quantitative traits, and its role in evolution (Steppan et al. 2002). However, the $\mathbf{G}$ matrix is not itself a representation of source laws of propensities of variation: instead, it is the matrix representing the contribution of new mutations, $\mathbf{M}$, that is most relevant to understanding the role of mutation and altered development in the evolution of quantitative characters. Houle (1998) has made some progress in developing a more 
mechanistic understanding of $\mathbf{M}$, based on how it is influenced by developmental-genetic factors such as mutational target size and timing of expression.

\section{Consequence laws of variation}

With respect to quantitative characters, consequence laws establish how $\mathbf{G}$ depends on $\mathbf{M}$, and in turn, how changes in trait values depend on $\mathbf{G}$, the latter of which is familiar from the canonical framework for the change in mean trait values, $\Delta \mathbf{z}=\mathbf{G} \mathbf{P}^{-1} \mathbf{S}$, where $\mathbf{P}$ is the matrix of phenotypic variances and covariances, and $\mathbf{S}$ is the vector of covariances of traits with fitness (Steppan et al. 2002). The dependence of $\mathbf{G}$ on $\mathbf{M}$ traditionally was ignored on the grounds that it is a small fraction of $\mathbf{G}$, nevertheless, the two are highly correlated, presumably because $\mathbf{G}$ reflects effects of $\mathbf{M}$ accumulated over many generations (Houle 1998).

With respect to discrete characters, there is no canonical framework to invoke as a source of consequence laws, though one can be sketched as follows, simply by applying existing theory to the case in which two different rates of mutation-and-altered-development are at work. In the case of discrete characters in an infinite space of possibilities, with rare mutations, evolutionary change will reach a steady-state rate of origin-fixation given by, for the haploid case:

$$
R=\mu N \pi
$$

where $\pi$ is the probability of fixation, and $\mu \mathrm{N}$ is the rate of origin (N, population size; $\mu$, mutation rate per individual; for the diploid case, replace $\mathrm{N}$ with $2 \mathrm{~N}$ ). The probability of fixation of a new allele, $\pi$, is given by (section 8.8.3 of Crow and Kimura 1970):

$$
\pi(s, p=1 / N)=\frac{1-e^{-2 s}}{1-e^{-2 N s}}
$$


Suppose, in the case of nucleotide characters, that the rate of mutation from a GC base pair to an AT base pair, $\mu_{\mathrm{i}}$, is higher by some factor B than the reverse rate $\mu_{\mathrm{j}}$, that is, $\mu_{\mathrm{i}} / \mu_{\mathrm{j}}=\mathrm{B}$. The GC-content in the third position of codons in diverse genomes ranges from about $11 \%$ to $90 \%$ (Singer and Hickey 2000), suggesting (according to the simplest possible interpretation) that B ranges from 0.1 to 10 . The rate of introduction of new alleles into the population is subject to this same bias, the consequence of which may be represented by partitioning the overall rate of evolution into the expected rates due to $\mathrm{GC} \rightarrow \mathrm{AT}$ changes and to $\mathrm{AT} \rightarrow \mathrm{GC}$ changes, and then considering their ratio:

$$
\frac{R_{i}}{R_{j}}=\frac{\mu_{i} N \pi_{i}}{\mu_{j} N \pi_{j}}=B \frac{\pi_{i}}{\pi_{j}}
$$

The neutral case of this model, in which B is the only effect, is the implicit basis for much interpretation of systematic differences in GC content among genomes (Lobry 1997). From this consequence law, for instance, it follows that in the long term, sequence composition will change by neutral evolution until the GC:AT ratio is equal to B (Sueoka 1993).

Molecular evolutionists often assume that only neutral evolution can be biased in this manner, but this notion has no basis, as explained earlier. The probability of fixation for beneficial mutations is $\pi(\mathrm{s}) \approx 2 \mathrm{~s}$ where $0<\mathrm{s}<<1$, thus from Eqn 3, the bias in rates of beneficial changes (given the same assumptions), is:

$$
\frac{R_{i}}{R_{j}} \approx \frac{\mu_{i} N * 2 s_{i}}{\mu_{j} N * 2 s_{j}}=B \frac{s_{i}}{s_{j}}
$$

For instance, if $\mathrm{B}=10$, but beneficial $\mathrm{GC} \rightarrow \mathrm{AT}$ changes are only half as beneficial as $\mathrm{AT} \rightarrow \mathrm{GC}$ changes, then the bias in rates is no longer 10 , but $10 / 2=5$. If the advantage of beneficial 
$\mathrm{GC} \rightarrow \mathrm{AT}$ changes is not different from that of beneficial AT $\rightarrow \mathrm{GC}$ changes, then $\mathrm{s}_{\mathrm{i}}=\mathrm{s}_{\mathrm{j}}$ and the bias in the rate of evolution is $\mathrm{B}$, as for the neutral case.

Infinite spaces and long-term effects, though useful for considering sequence divergence, are not very reasonable in other cases, thus it is helpful to consider the short-term consequence of a bias in variation in terms of what may be called an "option coefficient" or coefficient of preference, characterizing the probability that the next step in evolution is a step of one type rather than another, or in this case, a $\mathrm{GC} \rightarrow \mathrm{AT}$ change as opposed to an $\mathrm{AT} \rightarrow \mathrm{GC}$ change. This option coefficient is equivalent to the bias in rates (Eqn 3 or 4) when origin-fixation events are sufficiently rare that they do not interfere with each other. For instance, starting with a sequence with equal frequencies of $\mathrm{GC}$ and $\mathrm{AT}$, the chance that the next change is a $\mathrm{GC} \rightarrow \mathrm{AT}$ change as opposed to an $\mathrm{AT} \rightarrow \mathrm{GC}$ change is $\mathrm{Bs}_{\mathrm{i}} / \mathrm{s}_{\mathrm{j}}$, that is, $\mathrm{Bs}_{\mathrm{i}} / \mathrm{s}_{\mathrm{j}}$ is the coefficient of preference for $\mathrm{GC} \rightarrow \mathrm{AT}$ over $\mathrm{AT} \rightarrow \mathrm{GC}$. The same consequence law, Eqn 4, holds approximately (given small $\mu \mathrm{N}$ ) for the choice between two adaptive peaks in the population model of Yampolsky and Stoltzfus (2001).

The linear dependence of an option coefficient on B is not necessarily limited to the case in which change is initiated by an event of mutation. To consider evolution from standing variation, we may begin with a more general form of the probability of fixation that includes an initial frequency, p (section 8.3.3 of Crow and Kimura 1970):

$$
\pi(s, p)=\frac{1-e^{-2 N s p}}{1-e^{-2 N s}}
$$

Consider the case in which the population includes deleterious alleles in mutation-selection balance, some of which become advantageous upon a change in environment. The expected 
starting frequency of each allele is then $p=\mu / s_{d}$ (see section 6.2 of Crow and Kimura 1970), where $s_{d}$ is the selective disadvantage. Substituting $p=\mu / s_{d}$ into Eqn 5 yields the probability that an allele in standing variation will be fixed when conditions change so that it now has an advantage $\mathrm{s}_{\mathrm{b}}$ :

$$
\mathrm{P}\left(s_{b}, s_{d}\right)=\frac{1-e^{-2 N s_{b} \frac{\mu}{s_{d}}}}{1-e^{-2 N s_{b}}} \cong 2 N \frac{s_{b}}{s_{d}} \mu
$$

when $2 \mathrm{~N} \mu \mathrm{s}_{\mathrm{b}} / \mathrm{s}_{\mathrm{d}}$ is small (compare Eqn 3 of Orr and Betancourt 2001). Since this is linearly dependent on $\mu$, the option coefficient for choosing between two types of variants that emerge with rates $\mu_{\mathrm{i}}$ and $\mu_{\mathrm{j}}$ is linearly dependent on the ratio $\mathrm{B}=\mu_{\mathrm{i}} / \mu_{\mathrm{j}}$. This ignores any effect of competition or linkage between multiple beneficial alleles present simultaneously (for a partial treatment of such effects, see Gerrish and Lenski 1998).

Finally, and most importantly, there is no need to limit our attention to the case of GC $\rightarrow$ AT bias, or any other mutational bias, as opposed to asymmetries in the emergence of variant phenotypes imposed by the mechanics of development. To understand this point, we recast Eqn 4 as a ratio of sums, each covering a class of changes:

$$
\frac{R_{i}}{R_{j}} \cong \frac{\sum_{i} 2 N \mu_{i} s_{i}}{\sum_{j} 2 N \mu_{j} s_{j}}=\frac{\sum_{i} \mu_{i} s_{i}}{\sum_{j} \mu_{j} s_{j}}
$$

In this manner, we may consider the option coefficient for any arbitrary set of alternative states $i$ relative to any other arbitrary set $j$, where the sets may be defined in terms of different types of mutations, different directions in morphospace, different sizes of effects, etc. A special case of Eqn 7 would be the chance of choosing one alternative state $i$ of interest, relative to the set $j$ of 
all possible alternative states, as in the equation used by Orr (2002) to choose the next step in an adaptive walk (though Orr assumes that mutation has no biases).

In particular, we may wish to consider the option coefficient for choosing some phenotype $\mathrm{P}_{\mathrm{i}}$ mapping to a set of mutationally accessible genetic states $G_{i}$, relative to some other phenotype $P_{j}$ corresponding to genotypes $\mathrm{G}_{\mathrm{j}}$. If the size of one set of alternative genetic states is larger than the other, for instance, if it corresponds to a developmentally favored phenotype according to the second conjecture of Alberch and Gale, then this category of changes will be favored, other things being equal. The simplest application of this kind of argument would be King's argument regarding the genetic code, which relies on the fact that different amino acids correspond to different volumes of the mutationally accessible genotype space of codons. Likewise, Yampolsky and Stoltzfus (2001) give an intuitive argument that the influence of the bias factor B in their population model would be the same whether it is a mutation bias per se, or a developmental bias, given a definition of developmental bias in terms of the numbers of alternative genotypes assigned to two alternative phenotypes.

The above considerations are not intended to be definitive, but merely to suggest a formal basis for the claim that - whether one is considering genotypes or phenotypes, neutral or adaptive evolution, new mutations or standing variation - a bias in the emergence of variation can be expected to impose a bias on the course of evolution.

\section{Synopsis}

Darwin's mechanism of hereditary change was discarded a century ago. What unites the Modern Synthesis with Darwin's theory, and distinguishes both from mutationism, is adherence to the 
doctrines that evolutionary change is composed of infinitesimal increments ("gradualism"), and that selection is the sole or overwhelming source of creativity, directionality, and initiative (dynamics) in evolution. The architects of the Modern Synthesis claimed that these doctrines follow (via the "gene pool") from the genetics of reproducing organisms, and that they are consistent with the observed behavior of evolution. The mutationists argued that change often is not composed of infinitesimal increments, and that mutation-and-altered-development is a source of discontinuity, directionality, creativity and initiative. The Modern Synthesis was adopted and became the foundation of subsequent evolutionary thought.

Nevertheless, it is now clear that this consensus was premature. Subsequent theoretical research has shown that the principles of genetics do not guarantee the validity of Darwinian doctrines. Instead, mutation-and-altered-development is a possible cause of discontinuity, directionality and dynamics. Subsequent empirical research has shown that evolutionary change is not always composed of infinitesimal increments, and that the character and dynamics of variation influence the rate and direction of evolutionary change.

What is most important to understand about these results is that they demand a new and unfamiliar way of understanding evolutionary causation, including ideas that, due to the rhetorical excesses of the Modern Synthesis, are likely to be seen as heresies or conceptual errors. In particular, evolutionary developmental biologists have been told repeatedly that development cannot be construed as an evolutionary cause, but this restriction only applies within the Modern Synthesis. Within a more general theory, it is possible to articulate a formal basis for recognizing mutational and developmental propensities in the generation of variation as a cause of non-randomness in the form of parallelisms, persistent patterns, or directional trends. 
This theoretical basis depends first, on recognizing the introduction of variants by mutationand-altered-development as a causal process within evolution (not a pre-condition for "evolution") and second, on recognizing propensities in the introduction of variation that may impose propensities on evolution.

None of this should be taken as a call to return to the mutationism of the 1920's or to abandon the adaptationist research program. The recollection of mutationism here serves mainly as a timely reminder that the Modern Synthesis is not the theory of evolution, but $a$ theory of evolution. While the adaptationist research program may continue building on its success in attributing non-randomness to selection, a new methodological imperative is warranted, to identify and quantify propensities of variation, and to assess their impact on evolutionary change.

\section{Acknowledgements}

Dan Buchholz, Eric Haag, Norman Johnson, Alec Panchen, John True, and an anonymous reviewer are thanked for their comments. Supporting material (with complete quotations) is available online at http://www.molevol.org/camel/publications/s06ed.

\section{Literature Cited}

Alberch, P. 1982. Developmental Constraints in Evolutionary Processes. In Evolution and Development. (ed. J.T. Bonner). Springer-Verlag, New York.

Alberch, P., and Gale, E.A. 1985. A developmental analysis of an evolutionary trend: digital reduction in amphibians. Evolution 39: 8-23. 
Ancel, L.W., and Fontana, W. 2000. Plasticity, evolvability, and modularity in RNA. J Exp Zool 288: 242-283.

Arthur, W. 2000. The concept of developmental reprogramming and the quest for an inclusive theory of evolutionary mechanisms. Evolution and Development 2: 49-57.

Arthur, W. 2004. Biased Embryos and Evolution. Cambridge University Press, Cambridge, pp. 248.

Ayala, F.J. 1970. Teleological Explanations in Evolutionary Biology. Philosophy of Science 37: $1-15$.

Ayala, F.J., and Fitch, W.M. 1997. Genetics and the origin of species: an introduction. Proc Natl Acad Sci U S A 94: 7691-7697.

Barton, N., and Partridge, L. 2000. Limits to natural selection. Bioessays 22: 1075-1084.

Bateson, W. 1894. Materials for the Study of Variation, Treated with Especial Regard to Discontinuity in the Origin of Species. Macmillan, London, pp. 598.

Beletskii, A., and Bhagwat, A.S. 1996. Transcription-induced mutations: increase in C to T mutations in the nontranscribed strand during transcription in Escherichia coli. Proc Natl Acad Sci U S A 93: 13919-13924.

Bell, G. 1997. Selection: The Mechanism of Evolution. Chapman and Hall, New York, pp. 688.

Berry, R.J. 1982. Neo-Darwinism. Edward Arnold, Ltd., London, pp. 68.

Bull, J.J., and Otto, S.P. 2005. The first steps in adaptive evolution. Nat Genet 37: 342-343. 
Chetverikov, S.S. 1997. On Certain Aspects of the Evolutionary Process from the Standpoint of Modern Genetics. Genetics Heritage Press, Placitas, New Mexico, pp. 100.

Cheverud, J.M. 1988. A Comparison of Genotypic and Phenotypic Correlations. Evolution 42: 958-968.

Cronin, H. 1991. The Ant and the Peacock. Cambridge University Presss, Cambridge, pp. 490.

Crow, J.F., and Kimura, M. 1970. An Introduction to Population Genetics Theory. Burgess, Minneapolis, pp. 591.

Dagan, T., Talmor, Y., and Graur, D. 2002. Ratios of radical to conservative amino acid replacement are affected by mutational and compositional factors and may not be indicative of positive Darwinian selection. Mol Biol Evol 19: 1022-1025.

Darwin, C. 1859. On the Origin of Species, 1st ed. John Murray, London.

Dawkins, R. 1987. The Blind Watchmaker. W.W. Norton and Company, New York, pp. 332.

Dawkins, R. 1996. Climbing Mount Improbable. W.W. Norton and Company, New York, pp. 340.

De Vries, H. 1905. Species and Varieties: Their Origin by Mutation. The Open Court Publishing Company, Chicago, pp. 847.

Dobzhansky, T. 1955. Genetics and the Origin of Species. Wiley \& Sons, Inc., New York, pp. 364. 
Dobzhansky, T., Ayala, F.J., Stebbins, G.L., and Valentine, J.W. 1977. Evolution. W.H. Freeman, pp. 571.

Eimer, T. 1898. On Orthogenesis; and The Impotence of Natural Selection in SpeciesFormation. Open Court Publishing Co., Chicago.

Eldredge, N. 2001. The Triumph of Evolution and the Failure of Creationism. W H Freeman \& Co, pp. 224.

Fisher, R.A. 1930. The Genetical Theory of Natural Selection. Oxford University Press, London.

Ford, E.B. 1971. Ecological Genetics, 3rd ed. Chapman \& Hall, London, pp. 410.

Freese, E. 1962. On the Evolution of the Base Composition of DNA. J. Theoret. Biol. 3: 82-101.

Fryxell, K.J., and Zuckerkandl, E. 2000. Cytosine deamination plays a primary role in the evolution of mammalian isochores. Mol Biol Evol 17: 1371-1383.

Galtier, N., and Lobry, J.R. 1997. Relationships between genomic G+C content, RNA secondary structures, and optimal growth temperature in prokaryotes. J Mol Evol 44: 632-636.

Gerrish, P.J., and Lenski, R.E. 1998. The fate of competing beneficial mutations in an asexual population. Genetica 103: 127-144.

Gillespie, J.H. 1984. Molecular Evolution over the Mutational Landscape. Evolution 38: 11161129.

Goldman, N., and Yang, Z. 1994. A codon-based model of nucleotide substitution for proteincoding DNA sequences. Mol Biol Evol 11: 725-736. 
Goldschmidt, R. 1940. The Material Basis of Evolution. Yale University Press, New Haven, pp. 436.

Gould, S.J. 1977. Ever Since Darwin. W.W. Norton \& Co., New York, pp. 285.

Gould, S.J. 2002. The Structure of Evolutionary Theory. Harvard University Press, Cambridge, Massachusetts, pp. 1464.

Haldane, J.B.S. 1932. The Causes of Evolution. Longmans, Green and Co., New York, pp. 235.

Hartl, D.L., and Taubes, C.H. 1998. Towards a theory of evolutionary adaptation. Genetica 103: 525-533.

Hill, W.G. 1982. Rates of change in quantitative traits from fixation of new mutations. Proc Natl Acad Sci U S A 79: 142-145.

Houle, D. 1998. How should we explain variation in the genetic variance of traits? Genetica 102103: 241-253.

Houle, D., Morikawa, B., and Lynch, M. 1996. Comparing mutational variabilities. Genetics 143: 1467-1483.

Hull, D.L. 2002. History of Evolutionary Thought. In Encyclopedia of Evolution. (ed. M. Pagel), pp. E7-E16. Oxford University Press, New York.

Huxley, J.S. 1942. Evolution: The Modern Synthesis. George Allen \& Unwin, London. 
Hwang, D.G., and Green, P. 2004. Bayesian Markov chain Monte Carlo sequence analysis reveals varying neutral substitution patterns in mammalian evolution. Proc Natl Acad Sci US A 101: 13994-14001.

Kimura, M. 1983. The Neutral Theory of Molecular Evolution. Cambridge University Press, Cambridge, pp. 367.

King, J.L. 1971. The Role of Mutation in Evolution. In Sixth Berkeley Symposium on Mathematical Statistics and Probability. (eds. L.M. Le Cam, J. Neyman, and E.L. Scott), pp. 69-88. University of California Press, Berkeley, California.

Lewontin, R.C. 1965. The Gene and Evolution. In Mendel Centenary: Genetics, Development and Evolution. (ed. R.M. Nardone), pp. 67-75. Catholic University of America Press, Washington, DC.

Li, W.-H. 1997. Molecular Evolution. Sinauer, Sunderland, Mass., pp. 487.

Lobry, J.R. 1997. Influence of genomic $\mathrm{G}+\mathrm{C}$ content on average amino-acid composition of proteins from 59 bacterial species. Gene 205: 309-316.

Mani, G.S., and Clarke, B.C. 1990. Mutational order: a major stochastic process in evolution. Proc R Soc Lond B Biol Sci 240: 29-37.

Maynard Smith, J. 1975. The Theory of Evolution, 3rd ed. Cambridge University Press, Cambridge.

Maynard Smith, J. 1983. Evolution and Development. In Development and Evolution. (eds. B.C. Goodwin, N. Holder, and C.C. Wylie), pp. 33-46. Cambridge University Press, New York. 
Maynard Smith, J., Burian, R., Kauffman, S., Alberch, P., Campbell, J., Goodwin, B., Lande, R., Raup, D., and Wolpert, L. 1985. Developmental Constraints and Evolution. Quart. Rev. Biol. 60: 265-287.

Mayr, E. 1960. The Emergence of Evolutionary Novelties. In Evolution After Darwin: The University of Chicago Centennial. (eds. S. Tax, and C. Callender), pp. 349-380. University of Chicago Press, Chicago.

Mayr, E. 1963. Animal Species and Evolution. Harvard University Press, Cambridge, Massachusetts, pp. 797.

Mayr, E. 1980. Some Thoughts on the History of the Evolutionary Synthesis. In The Evolutionary Synthesis. (eds. E. Mayr, and W. Provine), pp. 1-48. Harvard University Press, Cambridge, MA.

Mayr, E. 1983. How to carry out the adaptationist program? Am. Nat. 121: 324-334.

Mayr, E. 1994a. The Resistance to Darwinism and the Misconceptions on which it was Based. In Creative Evolution? (eds. J.H. Campbell, and J.W. Schopf), pp. 35-46. Jones \& Bartlett, Inc., London.

Mayr, E. 1994b. Response to John Beatty. Biology and Philosophy 9: 357-358.

Medawar, P.B. 1967. The Art of the Soluble. Methuen and Co., London, pp. 160.

Mooers, A.O., and Holmes, E.C. 2000. The evolution of base composition and phylogenetic inference. Trends in Ecology and Evolution 15: 365-369. 
Morgan, T.H. 1903. Evolution and Adaptation. Macmillan, New York.

Morgan, T.H. 1916. A Critique of the Theory of Evolution. Princeton University Press, Princeton, NJ, pp. 197.

Morgan, T.H. 1925. Evolution and Genetics, Second ed. Princeton University Press, Princeton, pp. 211.

Muller, H.J. 1923. Mutation. Eugenics, Genetics and the Family 1: 106-112.

Muller, H.J. 1956. On the Relation between Chromosome Changes and Gene Mutation. Brookhaven Symposia in Biology 8: 126-147.

Muse, S.V., and Gaut, B.S. 1994. A likelihood approach for comparing synonymous and nonsynonymous nucleotide substitution rates, with application to the chloroplast genome. Mol Biol Evol 11: 715-724.

Nei, M. 1987. Molecular Evolutionary Genetics. Columbia University Press, New York, pp. 512.

Orr, H.A. 1999. The evolutionary genetics of adaptation: a simulation study. Genet Res 74: $207-$ 214.

Orr, H.A. 2002. The population genetics of adaptation: the adaptation of DNA sequences. Evolution Int J Org Evolution 56: 1317-1330.

Orr, H.A. 2005. The genetic theory of adaptation: a brief history. Nat Rev Genet 6: 119-127.

Orr, H.A., and Betancourt, A.J. 2001. Haldane's sieve and adaptation from the standing genetic variation. Genetics 157: 875-884. 
Orr, H.A., and Coyne, J.A. 1992. The Genetics of Adaptation: A Reassessment. American Naturalist 140: 725-742.

Petrov, D.A., and Hartl, D.L. 1999. Patterns of nucleotide substitution in Drosophila and mammalian genomes. Proc Natl Acad Sci U S A 96: 1475-1479.

Phillips, P.C. 1996. Waiting for a compensatory mutation: phase zero of the shifting-balance process. Genet Res 67: 271-283.

Punnett, R.C. 1911. Mendelism, 3rd ed. MacMillan, pp. 192.

Punnett, R.C. 1930. Genetics, Mathematics, and Natural Selection. Nature 126: 595-597.

Reeve, H.K., and Sherman, P.W. 1993. Adaptation and the Goals of Evolutionary Research. Quarterly Review of Biology 68: 1-32.

Ridley, M. 1985. The Problems of Evolution. Oxford University Press, Oxford, pp. 159.

Ridley, M. 2002. Natural Selection: An Overview. In Encyclopedia of Evolution. (ed. M. Pagel), pp. 797-804. Oxford University Press, New York.

Rokyta, D.R., Joyce, P., Caudle, S.B., and Wichman, H.A. 2005. An empirical test of the mutational landscape model of adaptation using a single-stranded DNA virus. Nat Genet 37: 441-444.

Schluter, D. 1996. Adaptive Radiation Along Genetic Lines of Least Resistance. Evolution 50: 1766-1774.

Shull, A.F. 1935. Weismann and Haeckel: One Hundred Years. Science 81: 443. 
Shull, A.F. 1936. Evolution, 1st ed. McGraw-Hill, New York, pp. 312.

Simpson, G.G. 1967. The Meaning of Evolution, 2nd ed. Yale University Press, New Haven, Conn., pp. 368.

Singer, G.A., and Hickey, D.A. 2000. Nucleotide Bias Causes a Genomewide Bias in the Amino Acid Composition of Proteins. Mol Biol Evol 17: 1581-1588.

Sober, E. 1984. The Nature of Selection: Evolutionary Theory in Philosophical Focus. MIT Press, Cambridge, Mass., pp. 400.

Stebbins, G.L. 1966. Processes of Organic Evolution. Prentice Hall, Englewood Cliffs, NJ, pp. 191.

Stebbins, G.L. 1982. Darwin to DNA, Molecules to Humanity. W.H. Freeman and Company, San Francisco, pp. 491.

Steppan, S.J., Phillips, P.C., and Houle, D. 2002. Comparative Quantitative Genetics: Evolution of the G Matrix. Trends in Ecology and Evolution 17: 320-327.

Stoltzfus, A. 1999. On the possibility of constructive neutral evolution. J Mol Evol 49: 169-181.

Sueoka, N. 1962. On the Genetic Basis of Variation and Heterogeneity of DNA Base Composition. Proc. Natl. Acad. Sci. U.S.A. 48: 582-592.

Sueoka, N. 1993. Directional mutation pressure, mutator mutations, and dynamics of molecular evolution. J Mol Evol 37: 137-153. 
Thomson, K.S. 1985. Essay review: the relationship between development and evolution. Oxford Surveys in Evolutionary Biology 2: 220-233.

Vavilov, N.I. 1922. The Law of Homologous Series in Variation. J. Heredity 12: 47-89.

Vrba, E.S., and Eldredge, N. 1984. Individuals, hierarchies and processes: towards a more complete evolutionary theory. Paleobiology 10: 146-171.

Yampolsky, L.Y., and Stoltzfus, A. 2001. Bias in the introduction of variation as an orienting factor in evolution. Evol Dev 3: 73-83.

Yampolsky, L.Y., and Stoltzfus, A. 2005. Untangling the effects of codon mutation and amino acid exchangeability. Pac Symp Biocomput: 433-444.

Zhang, Z., and Gerstein, M. 2003. Patterns of nucleotide substitution, insertion and deletion in the human genome inferred from pseudogenes. Nucleic Acids Res 31: 5338-5348. 